\title{
Design of Video Teaching System Based on Virtual Reality Technology
}

\author{
Song Wang*, Hanwen Zhang, Xiang Meng
}

Xiangshan Film and Television College, Ningbo University of Finance \& Economics, Ningbo 315175, China. E-mail: 47499510@qq.com

\begin{abstract}
With the progress of the times and the development of network technology, great changes have taken place in the mode of education and teaching, and the traditional mode of education and teaching has been unable to meet the current teaching situation and requirements. Under this background, many scholars have developed a variety of teaching models adapted to the background of the times, which has achieved good results. In order to realize the reform and innovation of teaching mode, this paper designs a film and television teaching system based on virtual reality technology. In the era of Internet of Things and big data, Internet of Things technology is used as support to realize the transmission and sharing of teaching information in film and television teaching system. In the research, data mining technology is used to collect relevant data of teaching resources and teaching information, and relevant algorithms in data mining are used to realize data processing. Experiment of film and television teaching system in different stages of education system and the application of film and television teaching theory courses, experiment courses for students and education development index are analyzed. According to the end of the experiment, the influence of results show that the film and television teaching system in the use of elementary school, junior high school, high school and university level are $25.32 \%, 31.44 \%, 18.46 \%$ and $47.76 \%$ respectively. The film and television teaching system plays a significant role in students' experimental course scores. The use of the film and television teaching system has raised the development index of education and teaching in each stage of education from 1.12, 1.33, 1.47 and 1.36 to 2.14, 2.21, 2.36 and 2.44 respectively, indicating that the film and television teaching system has a certain promoting effect on the development of education and teaching.
\end{abstract}

Keywords: Virtual Reality Technology; Internet of Things Technology; Data Mining Technology; Film and Television Teaching System

\section{Introduction}

\subsection{Research background}

In this era, as a part of education, the use of virtual reality technology for practical teaching has become a trend in the education industry. In fact, it can effectively solve the problem of funds and scenarios in traditional teaching practice and improve the awareness of resource allocation. Based on this, this research designed a film and television teaching system based on virtual reality technology to realize the reform and innovation of education and teaching. The film and television teaching system based on virtual reality technology can bring students a unique learning experience. The system uses virtual reality technology to simulate real scenes and

Copyright (C) 2020 Song Wang et al.

doi: $10.18686 /$ esta.v7i4.167

This is an open-access article distributed under the terms of the Creative Commons Attribution Non-Commercial License

(http://creativecommons.org/licenses/by-nc/4.0/), which permits unrestricted non-commercial use, distribution, and reproduction in any medium,

provided the original work is properly cited. 
provide students with a good teaching environment according to their needs. The system can also realize the interaction between students and things in the scene, and improve students' practical ability. The design and development of the film and television teaching system has very important scientific research value in education and teaching.

At present, many scholars have studied the teaching mode based on virtual reality technology. Zeming introduces a teaching system design and implementation of Korean. In order to improve the efficiency of teaching and students' interest in learning, the system uses the virtual reality technology, the results show that the virtual reality technology can change the traditional teaching mode, stimulate students' interest in learning, improve the teaching quality of Korean and Zeming's results will make the further application of virtual reality technology in the field of education ${ }^{[1]}$. Nisiforou took 37 primary school students as research objects, explored the education supply of virtual reality system as an auxiliary tool for foreign language teaching and students' cognition, and conducted assessment tests and questionnaire surveys on the participants. The results showed that students', performance had positive results because it encouraged them to visualize abstract knowledge in a virtual world. Nisiforou reveals the growing potential of virtual reality in teaching practice which can be used as a reference point for follow-up research. It also highlights the vision for a further agenda on the dynamic nature of virtual reality in foreign language education and discusses the application of virtual reality in the education system in Cyprus $^{[2]}$.

This research is carried out in the Internet of Things and big data environment, and provides a good network environment for system design and data transmission. In the research, data mining technology and related algorithms are used to collect and process the resources in education and teaching, which saves the time of data collection and processing and improves the efficiency of the experiment. This research uses virtual reality technology to realize the design of the film and television teaching system, which brings reform and innovation to the education and teaching mode, and plays an important role in the research of education and teaching.

\section{Method}

\subsection{Virtual reality technology}

Virtual reality technology is a comprehensive integrated technology including computer graphics human-computer interaction technology, detection technology and three-dimensional imaging. It uses computers to generate realistic three-dimensional vision, hearing and other sensations, so that people as participants can experience and interact with the virtual environment naturally through appropriate devices ${ }^{[3,4]}$. Virtual reality technology is gradually developed from simple sounds. The visualization and dynamic simulation in virtual reality technology are characterized by fantasy, interactivity and immersion ${ }^{[5]}$. Virtual reality technology and theories are gradually developed and widely used in education, teaching, medical and military fields. In education and teaching, virtual reality technology is an information or media technology that puts students in a virtual environment, allowing students to feel immersed in the virtual environment. They can observe things in the environment without being restricted by time and space, and feel things that cannot be experienced in the real world.

\subsection{Internet of Things technology}

Internet of Things technology is a kind of intelligent information network based on Internet and mobile communication network, it uses has the perception, transfer, and the computing power of physical devices, in accordance with the rules of the network communication protocol, all items addressing independently connected to the Internet, information communication, in order to realize the interconnection between equipment, perception, transmission, intelligent processing ${ }^{[6,7]}$. IoT of scale and the difference determines the diversification of IoT technology, an Internet application system design, form, function, application and management needs the support of various technologies, including: automatic perception, embedded and mobile communications, computer networks, data processing, key technology such as intelligent control, information security. The Internet of Things architecture can be composed of three layers: perception layer, network layer and application layer ${ }^{[8]}$. The perceptive layer of the Internet of Things is the foundation of the development and implementation of the Internet of Things. In this layer, short-distance data recognition and transmission of objects are mainly realized. The network layer is also called the transport layer. Its main purpose is 
to accurately transmit the information obtained by the detection layer and realize data exchange. The application layer is mainly for the organization and collection of perceptual data in the Internet of Things, and plays an important role in the Internet of Things.

\subsection{Data mining technology}

Data mining is the process of finding effective information in a large amount of data in a database. The process of data mining includes data preprocessing, data mining, data analysis and evaluation and knowledge expression. In essence, data mining is a process in which a rule or model can be discovered by adopting some methods according to a business requirement ${ }^{[9]}$. Data mining covers many fields including statistics, machine learning, pattern recognition and so on. Data mining technology contains many kinds of data processing algorithms, including statistical analysis, association rules, cluster analysis and decision tree analysis. These algorithms are divided into supervised learning and unsupervised learning. Data mining can also be used to describe the concept of an object and its related characteristics, and can also detect the existence of data exceptions in the database. In fact, it is to extract data patterns from preprocessed data through some specific algorithms and formulas, and then evaluate and analyze the data patterns with relevant technologies to represent valuable information in the data. Data mining combines statistical methods and fuzzy mathematics theory, and takes visualization technology to study the data in the database ${ }^{[10]}$. Data mining is a relatively hot and promising field. With the widespread application of data mining technology, it brings great convenience to all aspects of our life and work.

\subsection{Film and television teaching system}

Film and television teaching system is an emerging modern education and teaching mode, which refers to the application of combining teaching knowledge with film and television equipment by means of visual imaging and Internet of Things technology. On the basis of Internet of Things technology, the film and television teaching system extracts and stores the effective teaching information in the big data in the database of the film and television teaching system through data mining technology. The main objects of film and television teaching system are students and teachers, and the main application places are schools, training institutions and other educational and teaching institutions. The film and television teaching system is quite different from the traditional teaching method. The system uses virtual reality technology to enable students to learn in the virtual simulation scene. This will enable students to have a sense of immersion in learning, and students can also interact with the teaching content in the virtual scene, so as to exercise their practical ability. The film and television teaching system, to a great extent, alleviates teachers' teaching pressure, improves students' attention in the learning process, and improves students' ability of independent learning. At the same time, the use of film and television teaching system solves the problem of lack of educational resources, reduces the funds spent by schools on educational resources and relevant textbooks, and the use of Internet of Things technology and data mining technology can achieve good resource sharing function among schools.

\section{Experiment}

\subsection{Data collection}

This research first consulted the literature on virtual reality technology and film and television teaching in the past five years, and designed the experiments in this article by referring to the experimental research and data in related literature. Then use the Internet of Things technology and data mining methods to mine and collect relevant data in the big data environment, and use relevant algorithms to process the collected data.

\subsection{Experimental data processing}

Data mining technology generally uses correlation analysis and co-variance analysis to preprocess data. There are two event sets and $U$ and $V$ in the database. $u_{i}, v_{i}$ represent the $i$ event in the two event sets, $n$ is the number of events, $\sigma$ is the variance of the event, and $E$ is the expected value of the event. The correlation formula and co-variance formula of the two event sets can be expressed as:

$$
\begin{aligned}
& r_{U, V}=\frac{\sum\left(u_{i}-\bar{U}\right)\left(v_{i}-\bar{V}\right)}{n \sigma_{U} \sigma_{V}} \\
& \operatorname{Cov}_{U, V}=E(U V)-\bar{U} \bar{V}
\end{aligned}
$$


plified to:

$$
r_{U, V}=\frac{\operatorname{Cov}(U, V)}{n \sigma_{U} \sigma_{V}}
$$

Association rule algorithm is one of the most important data processing algorithms in data mining tech-

confidence $(U \rightarrow V)=P(U \mid V)=P(U \cup V) / P(U)$

The support formula for events in the association rule algorithm is as follows:

$$
\operatorname{Support}(U \rightarrow V)=P(U \cup V)
$$

The correlation algorithm of events in the association rule algorithm is as follows:

$$
r_{U, V}{ }^{\prime}=\frac{P(U \cup V)}{P(U) P(V)}=P(V \mid U) / P(V)
$$

\section{Result}

\subsection{Application situation of film teaching system}

With the application of reality and virtual technol- nology. Association rule algorithm usually uses probability analysis to calculate the confidence, support and relevance of the event, and process the data. The confidence formula of the event in the association rule algorithm is as follows:

ogy in the film and television teaching system, the film and television teaching system has been well developed, and its application in education and teaching has become more and more impressive. This experiment counts the usage rate of the film and television teaching system in elementary schools, junior high schools, high schools, universities and training institutions, and analyzes the development prospects of the film and television teaching system based on the user's total score of the system, the system's favorable rate, general evaluation and negative evaluation rate. The statistics of the related data of the film and television teaching system are shown in Table 1.

Table 1. Statistics on the application of the film and television teaching system

\begin{tabular}{|l|l|l|l|l|l|}
\hline & Utilization rate & Total score & $\begin{array}{l}\text { High praise } \\
\text { rate }\end{array}$ & $\begin{array}{l}\text { General evalu- } \\
\text { ation }\end{array}$ & Poor rating rate \\
\hline Primary & $25.32 \%$ & 9 & $57 \%$ & $22 \%$ & $9 \%$ \\
\hline Junior middle & $31.44 \%$ & 8 & $44 \%$ & $31 \%$ & $16 \%$ \\
\hline High school & $18.46 \%$ & 6 & $37 \%$ & $16 \%$ & $27 \%$ \\
\hline University & $47.76 \%$ & 8 & $41 \%$ & $21 \%$ & $13 \%$ \\
\hline Training agency & $21.11 \%$ & 5 & $23 \%$ & $43 \%$ & $20 \%$ \\
\hline
\end{tabular}

According to the data in Table 1, we can see that the current use rate of the film and television teaching system in elementary schools, high schools and educational institutions is relatively low, and the use rate in universities is the highest. In order to analyze the relevant data of the film and television teaching system more intuitively, we converted the data in the table into the form of a graph, and the final result is shown in Figure 1. 


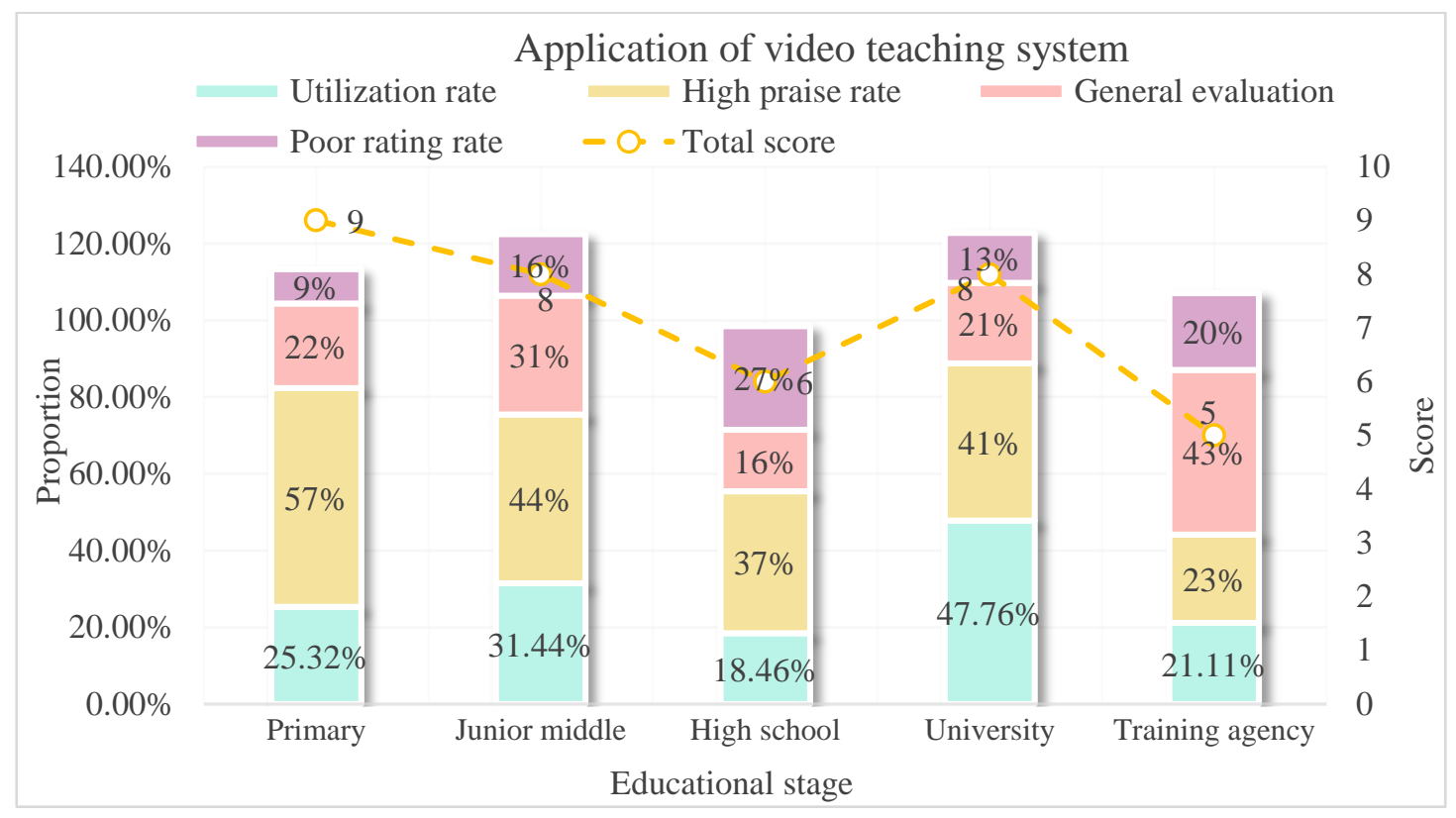

Figure 1. Application of the film and television teaching system.

According to the data in Figure 1, we can see that the use rate of the film and television teaching system in primary schools is only $25.32 \%$, but the film and television teaching system has the highest score, with a favorable rate of $57 \%$. The utilization rate of the video teaching system in junior high schools was $31.44 \%$, and the favorable rate was $44 \%$. High school has the lowest use rate of the film and television teaching system, only $18.46 \%$, and high school has the highest negative rate of the system, reaching $27 \%$. The utilization rate of the system by universities and training institutions was $47.76 \%$ and $21.11 \%$, and the favorable rate was $41 \%$ and $23 \%$. This shows that the development prospects of the film and television teaching system in elementary schools and universities are relatively good.

\subsection{Impact of the film and television teaching system}

The use of the video teaching system will have a certain impact on the development of education and teaching and the learning situation of students. This study counts the changes in the students' average scores of theoretical courses and experimental courses before and after using the film and television teaching system, and calculates the development index of education and teaching based on the experimental data, and analyzes the impact of the film and television teaching system on the development of education and teaching. The statistical results are shown in Table 2.

Table 2. Student performance changes and education and teaching development index table

\begin{tabular}{|l|l|l|l|l|l|l|}
\hline & \multicolumn{2}{|l|}{ Before use } & \multicolumn{2}{l|}{ After use } \\
\cline { 2 - 7 } & $\begin{array}{l}\text { Theoretical } \\
\text { achievements }\end{array}$ & $\begin{array}{l}\text { Experimental } \\
\text { results }\end{array}$ & Index & $\begin{array}{l}\text { Theoretical } \\
\text { achievements }\end{array}$ & $\begin{array}{l}\text { Experimental } \\
\text { results }\end{array}$ & Index \\
\hline Primary & 76 & 81 & 1.12 & 80 & 88 & 2.14 \\
\hline Junior middle & 81 & 84 & 1.33 & 84 & 90 & 2.21 \\
\hline High school & 89 & 88 & 1.47 & 92 & 96 & 2.36 \\
\hline University & 80 & 90 & 1.36 & 83 & 97 & 2.44 \\
\hline
\end{tabular}

According to the data in Table 2, we can see that the film and television teaching system has a certain positive effect on the students' theoretical and experimental course performance, and the film and television teaching system has a promoting effect on the development of education and teaching. In order to observe the data more intuitively, we convert the data in the table into a graph form, and the final result is shown in Figure 2. 


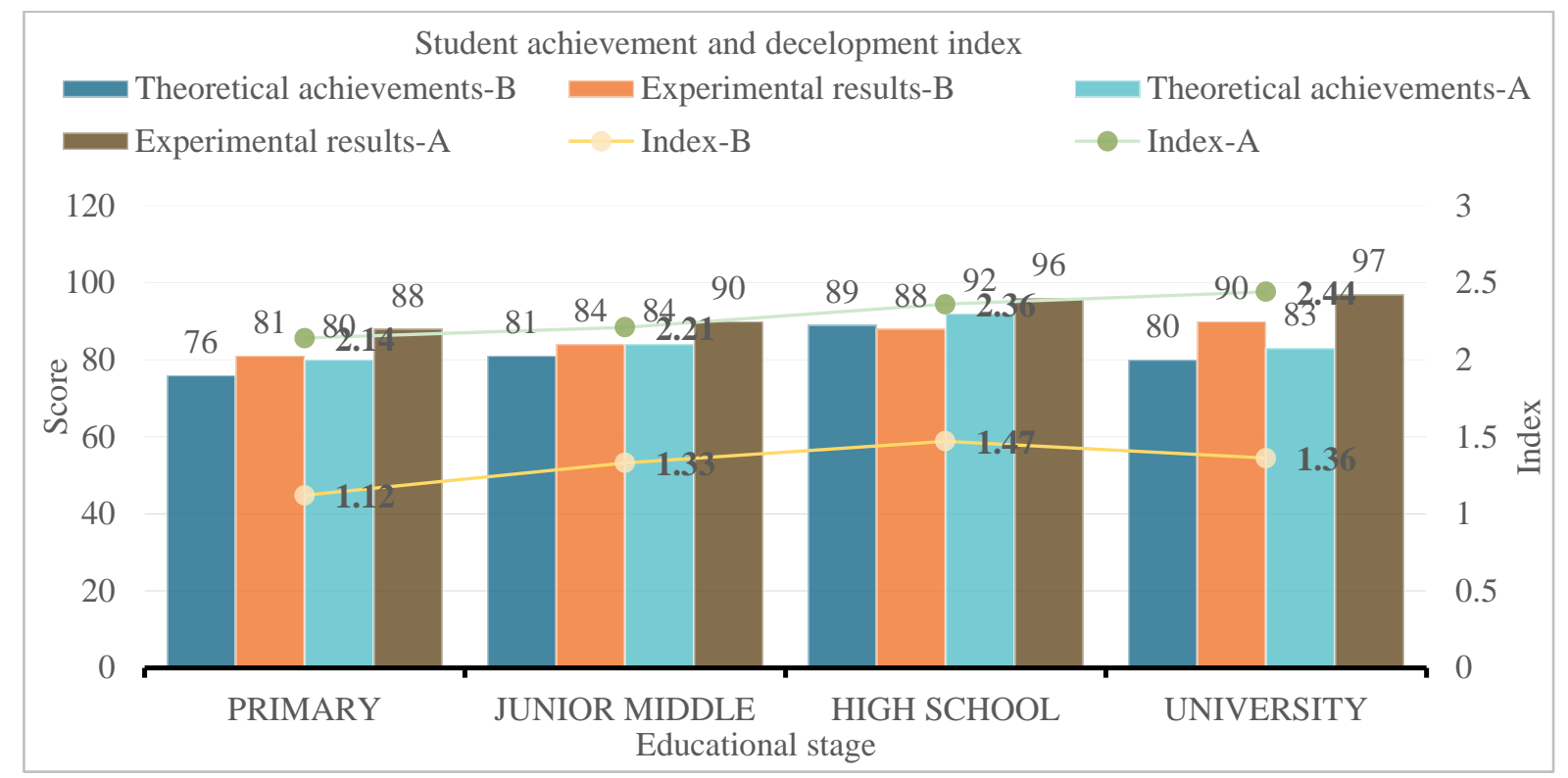

Figure 2. Student performance changes and educational development index graph.

According to the data in Figure 2, we can see that the film and television teaching system has a certain positive effect on the students' theoretical course performance and experimental course performance. The film and television teaching system has a relatively small effect on the students' theoretical course scores. The average score has only increased by about 5 points, and it has a significant effect on the students' experimental course scores. The average score has increased by about 8 points. The use of the film and television teaching system increases the educational and teaching development index of each stage of education from 1.12, 1.33, 1.47, and 1.36 to $2.14,2.21,2.36$, and 2.44 respectively, indicating that the film and television teaching system has a certain role in promoting the development of education and teaching.

\section{Conclusion}

In order to comply with the development of the education system, the reform and innovation of education and teaching models are imperative. The traditional education and teaching models can no longer meet the needs of students' learning and the development of education. Aiming at the reform and innovation of education and teaching, this paper uses virtual and reality technology to design a film and television teaching system, using the Internet of Things technology and the background of big data to realize the transmission and sharing of teaching resources. The film and television teaching system based on virtual reality technology has brought new reforms and innovations to the development of education, changing the traditional classroom teaching mode and teaching methods, providing students with suitable virtual classroom scenes and interactive virtual scenes function, exercising the students' practical ability.

\section{References}

1. Zeming L. Design and implementation of a Korean language teaching system based on virtual reality technology. Agro Food Industry Hi Tech 2017; 28(1): 2156-2159.

2. Nisiforou EFIA, Karageorgakis T. Virtual reality in the EFL classroom: Educational affordances and students' perceptions in Cyprus. Cyprus Review 2018; 30(1): 381-396.

3. Duan Y, Zhang J, Xie M, et al. Application of virtual reality technology in disaster medicine. Current Medical Science 2019; 39: 690-693.

4. Rousseaux F, Bicego A, Ledoux D, et al. Hypnosis associated with 3D immersive virtual reality technology in the management of pain: A review of the literature. Journal of Pain Research 2020; 13: 1129 1138.

5. Alfalah SFM. The value of virtual reality technology in embryology education; objective and subjective outcomes. International Journal of Psychosocial Rehabilitation 2020; 24(9): 4672-4684.

6. Rajeesh KN. Expending RSACQTT protocol for SDN based secure communication of Internet of Things. International Journal of Advanced Trends in Computer Science and Engineering 2020; 9(4): 5821-5827.

7. Kuncoro AH, Mellyanawaty M, Sambas A, et al. Air quality monitoring system in the city of Tasikmalaya based on the Internet of Things (IoT). 
Journal of Advanced Research in Dynamical and Control Systems 2020; 12(2): 2473-2479.

8. Singh R, Thakur A, Gehlot A. Internet of Things based on home automation for intrusion detection, smoke detection, smart appliance and lighting control. International Journal of Scientific \& Technology Research 2019; 8(12): 3702-3707.

9. Mayahi S, Yamchi A, Golalipour M, et al. Data mining for identification of forkhead box $\mathrm{O}$
(FOXO3a) in different organisms using nucleotide and tandem repeat sequences. Research in Molecular Medicine 2020; 8(1): 17-30.

10. Rezaianzadeh A, Dastoorpoor M, Sanaei M, et al. Predictors of length of stay in the coronary care unit in patient with acute coronary syndrome based on data mining methods. Clinical Epidemiology and Global Health 2020; 8(2): 383-388. 